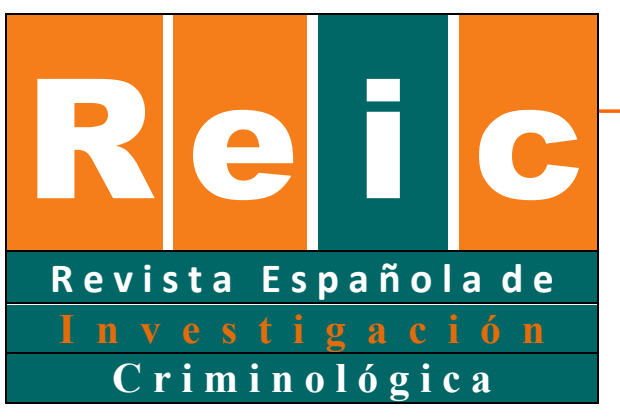

Ortega \& Jimeno

\title{
Revisión sistemática del concepto de polivictimización desde una perspectiva metodológica
}

\section{Systematic review of the concept of polyvictimization from a methodological perspective}

\author{
Beatriz Ortega Vidal iD \\ María Verónica Jimeno Jiménez ${ }^{1}$ \\ ${ }^{1}$ Departamento de Psicología. Centro de Investigación en Criminología. \\ Universidad de Castilla-La Mancha
}

\section{RESUMEN}

El concepto de polivictimización, entendido como la experimentación de múltiples tipos de victimización durante la infancia y la adolescencia, está ampliamente reconocido. El desarrollo del Cuestionario de Victimización Juvenil ha permitido un estudio comprehensivo de este fenómeno. No obstante, no hay acuerdo en la definición metodológica, existiendo múltiples elementos que inciden en la detección de polivíctimas. El objetivo es realizar una revisión del concepto de polivictimización desde una perspectiva metodológica. Se incluyen 30 artículos de los que se describe su método de análisis, el marco temporal analizado, el tipo de muestra y la versión del instrumento aplicada. Se discute cómo la variabilidad de criterios metodológicos construye una definición de polivíctimas diferente en cada investigación, afectando a los puntos de corte y a la detección de polivíctimas. Este trabajo ofrece orientación para comprender la prevalencia de la polivictimización, y puede servir como guía a nuevos investigadores de este campo de estudio.

Palabras clave: polivictimización, cuestionario de victimización juvenil, prevalencia, criterios metodológicos, infancia - adolescencia.

\footnotetext{
${ }^{1}$ La correspondencia debe dirigirse a: M. Verónica Jimeno Jiménez, veronica.jimeno@uclm.es
} 


\begin{abstract}
The concept of polyvictimization, understood as the experimentation of multiple types of victimization during childhood and adolescence, is widely recognized. The development of the Juvenile Victimization Questionnaire has allowed a comprehensive study of this phenomenon. However, there is no agreement on the methodological definition, with multiple elements affecting the detection of polyvictims. The aim is to carry out a review of the concept of polyvictimization from a methodological perspective. It includes 30 articles describing the method of analysis, the timeframe analyzed, the type of sample and the version of the instrument applied. It discusses how this variability of methodological critea build a different methodological definition of polyvictimization in each reasearch, affecting the cutoff points and the detection of polyvictims groups. This work offers guidance to understand the prevalence of polyvictimization and can serve as a guide for new researchers in this field of study.
\end{abstract}

Keywords: polyvictimization, juvenile victimization questionnaire, prevalence, methodological criteria, childhood - adolescence.

\title{
1. Introducción
}

Dentro del marco de la victimología del desarrollo, se ha producido en las últimas décadas un incremento en el estudio de la polivictimización infantil y juvenil, entendida esta como la experimentación de múltiples formas de victimización (Finkelhor, Ormrod, Turner y Hamby, 2005). Diversos estudios han puesto de manifiesto la alta exposición a diferentes formas de abuso y violencia que sufren los niños y adolescentes, así como el riesgo que supone la acumulación de experiencias traumáticas para la salud mental (Finkelhor, Ormrod, et al., 2005; Finkelhor, Ormrod y Turner, 2007a, 2007b; Turner, Finkelhor y Ormrod, 2006; Richmond, Elliott, Pierce, Aspelmeier y Alexander, 2009) y el comportamiento antisocial y delictivo (Ford, Elhai, Connor y Frueh, 2010), haciendo incidencia en la necesidad de considerar un amplio rango de victimizaciones a la hora de evaluar los perfiles de victimización de niños y adolescentes. Por ejemplo, se ha detectado una mayor incidencia de polivictimización entre los jóvenes inmersos en el sistema de justicia juvenil que en muestras comunitarias (Ford, Chapman, Connor y Cruise, 2012), teniendo además un mayor riesgo de suicidio, trastorno de estrés postraumático y consumo de alcohol y drogas que otros jóvenes institucionalizados (Croysdale, Drerup, Bewsey y Hoffmann, 2008; Ford, Grasso, Hawke y Chapman, 2013).

Revista Española de Investigación Criminológica

Artículo 6, Número 18 (2020)

https://doi.org/10.46381/reic.v18i1.399

www.criminologia.net

ISSN: 1696-9219 
El Cuestionario de Victimización Juvenil (JVQ o Juvenile Victimization Questionnaire; Hamby, Finkelhor, Ormrod y Turner, 2005), es un instrumento capaz de detectar un amplio rango de victimizaciones a través de un enfoque exhaustivo e integral. El JVQ cuenta con 34 ítems que se corresponden con diferentes tipos de victimización clasificadas en cinco módulos: delitos comunes, maltrato infantil, victimización por pares y/o hermanos, victimización sexual y victimización indirecta o haber sido testigo de violencia (Finkelhor, Hamby, Ormrod y Turner, 2005; Hamby et al., 2005; Hamby, Finkelhor, Turner y Kracke, 2011). El instrumento ha sido sometido a revisiones, siendo actualmente la segunda revisión, JVQ-R2, la que se encuentra disponible para su aplicación (Finkelhor, Hamby, Turner y Ormrod, 2011). En España, está disponible la adaptación del instrumento realizada por el Grupo de Investigación en Victimización Infantil y Adolescente (GReVIA) de la Universidad de Barcelona con el permiso de los autores (Pereda, Gallardo-Pujol y Guilera, 2018).

A pesar de que el concepto de polivictimización está ampliamente aceptado, no ocurre lo mismo con la definición metodológica (Pereda, 2019), coexistiendo tres métodos de análisis. El primer método considera polivíctimas a aquellas víctimas con una victimización por encima de la media de victimizaciones sufridas por el grupo (Finkelhor, Hamby et al., 2005). El segundo método define a las polivíctimas como aquellas que se encuentran dentro del 10\% superior más victimizado (Finkelhor, Ormrod y Turner, 2009). El tercer método hace referencia a las técnicas de agrupamiento, como el análisis por conglomerados tradicional (Holt, Finkelhor y Kaufman, 2007) o el análisis de clase latente (Ford et al., 2010; Ford, Grasso, Hawke y Chapman, 2013; Reid y Sullivan, 2009), utilizado para detectar perfiles de victimización similares, identificando a las víctimas con altos niveles de exposición a múltiples tipos de victimización.

Se ha analizado la capacidad que tienen los tres métodos para detectar al mismo grupo de polivíctimas y los resultados muestran que el grado de consistencia entre ellos es moderado (Segura, Pereda y Guilera, 2018). El debate sobre cuál es el mejor método para identificar a este grupo altamente victimizado aún está abierto (Pereda, 2019). Además, aspectos como el marco temporal, la versión del instrumento o las características de la

\section{Revista Española de Investigación Criminológica}

Artículo 6, Número 18 (2020)

https://doi.org/10.46381/reic.v18i1.399

www.criminologia.net

ISSN: 1696-9219 
muestra son elementos que, junto con el método de análisis, construyen esa definición (Segura et al., 2018).

Ante esta perspectiva, el presente trabajo tiene como objetivo realizar una revisión del constructo de polivictimización desde una perspectiva metodológica, describiendo los elementos que deben considerarse en la interpretación de su prevalencia.

\section{Método}

\subsection{Selección de los estudios}

Se realizó una búsqueda de estudios empíricos realizados durante los últimos nueve años (2010-2019) sobre polivictimización. Las bases de datos utilizadas fueron Scopus, ProQuest, Dialnet, EBSCO, Google Académico y Web Of Science. Las estrategias de búsqueda estuvieron compuestas por las palabras clave: polyvictimization, polivictimización, prevalence, prevalencia, juvenile victimization questionnaire, cuestionario de victimización juvenil, childhood, infancia, adolescence y adolescencia.

La búsqueda inicial dio como resultado una selección de 87 estudios potenciales, tras eliminar los duplicados y aquellos que no cumplían los criterios de inclusión, finalmente se incluyeron en la revisión 30 artículos. Cinco de ellos procedentes de fuentes secundarias.

Los criterios de inclusión fueron: (a) utilizar el Cuestionario de Victimización Juvenil como herramienta de recogida de información, (b) aportar evidencia empírica sobre la prevalencia de la polivictimización, (c) ser artículos de revistas científicas, (d) estar escritos en inglés o castellano y (e) haber sido publicado desde el 2010, éste inclusive.

\subsection{Características de los estudios}

En la Tabla 1 se recogen datos de los estudios seleccionados que se consideran relevantes para la revisión: (a) autores y año de publicación, (b) país en el que se ha desarrollado el estudio, (c) número de participantes, (d) tipo de muestra, (e) edad de los participantes, (f) método de análisis usado para detectar la polivictimización (víctimas por encima de la media,

\section{Revista Española de Investigación Criminológica}

Artículo 6, Número 18 (2020)

https://doi.org/10.46381/reic.v18i1.399

www.criminologia.net

ISSN: 1696-9219 
$10 \%$ superior más victimizado y técnicas de agrupamiento), (g) periodo de tiempo analizado (periodo de vida o último año de vida) y (h) versión del instrumento.

\section{Resultados}

La definición de polivictimización está sujeta a diferentes aspectos metodológicos entre los que se citan: el método de análisis utilizado, el periodo de tiempo sobre el que se mide la victimización, las características de la muestra y la versión del instrumento aplicada (Segura et al., 2018). Por lo que, al hablar de prevalencias y al interpretar estas cifras en los distintos estudios, es necesario prestar atención a estos aspectos metodológicos.

\subsection{Método de análisis y marco temporal}

El primer método propuesto por Finkelhor, Ormrod, Turner y Hamby (2005) considera polivíctimas a aquellas personas que tienen al menos una victimización por encima de la media de victimizaciones sufridas por el grupo durante su último año de vida. Este método permite realizar una clasificación en la que son víctimas aquellas que han sufrido entre 1 y 3 tipos de victimización, polivíctimas de nivel bajo aquellas que han sufrido entre 4 y 6 tipos, y polivíctimas de nivel alto las que han sufrido 7 o más tipos.

Mediante este método, el porcentaje de los niños, niñas y adolescentes polivíctimas varía desde un 8\% en Canadá (Cyr et al., 2013) y un 9.5\% en China (Chan, 2013), a cifras más elevadas en lugares como Chile, con un 37\% (Pinto-Cortez, Pereda y Álvarez-Lister, 2017), o en España, donde oscilan desde un 20\% (Pereda, Guilera y Abad, 2014) y un 45.2\% (Segura, Pereda, Abad y Guilera, 2015), llegando a un 61.4\% (Pereda, Abad y Guilera, 2017).

Revista Española de Investigación Criminológica

Artículo 6, Número 18 (2020)

https://doi.org/10.46381/reic.v18i1.399

www.criminologia.net

ISSN: 1696-9219 


\section{Tabla 1.}

Estudios incluidos en la revisión y sus características metodológicas

\begin{tabular}{|c|c|c|c|c|c|c|c|}
\hline Autores y año & País & $\mathbf{N}$ & Tipo de muestra & Edad & Método & Periodo & Versión del cuestionario \\
\hline $\begin{array}{l}\text { Pinto-Cortez, Flores- Jara, } \\
\text { Pereda y Guerra (2019) }\end{array}$ & Chile & 667 & Comunitaria & $12-17$ & $10 \%$ superior & Periodo de vida & $\begin{array}{l}\text { Adaptación } \quad \text { versión } \\
\text { española al lenguaje local }\end{array}$ \\
\hline $\begin{array}{l}\text { Segura, Pereda y Guilera } \\
\text { (2018) }\end{array}$ & España & 1.105 & Comunitaria & $12-17$ & $\begin{array}{l}\text { Víctimas por encima de la media } \\
10 \% \text { superior } \\
\text { Análisis de clase latente }\end{array}$ & $\begin{array}{l}\text { Periodo de vida } \\
\text { Último año }\end{array}$ & Adaptación española \\
\hline $\begin{array}{l}\text { Pinto-Cortez, Guitérrez- } \\
\text { Echegoyen y Henríquez } \\
(2018)\end{array}$ & Chile & 718 & Comunitaria & $17-28$ & $\begin{array}{l}\text { Víctimas por encima de la media } \\
10 \% \text { superior }\end{array}$ & Periodo de vida & Versión retrospectiva \\
\hline $\begin{array}{l}\text { Álvarez-Lister, } \begin{array}{r}\text { Pereda, } \\
\text { Guilera, Abad y Segura } \\
(2017)\end{array} \\
\end{array}$ & España & 472 & Clínica y comunitaria & $12-17$ & Víctimas por encima de la media & Último año & Adaptación española \\
\hline Cyr et al. (2017) & Canadá & 1.400 & Comunitaria & $12-17$ & Víctimas por encima de la media & Periodo de vida & Versión original \\
\hline $\begin{array}{l}\text { Indias-García y De Paúl- } \\
\text { Ochotorena (2017) }\end{array}$ & España & 608 & Comunitaria & $12-18$ & $10 \%$ superior & Periodo de vida & Adaptación española \\
\hline $\begin{array}{l}\text { Guerra, Inostroza, } \\
\text { Villegas, Villalobos y } \\
\text { Pinto-Cortez (2017) }\end{array}$ & Chile & 180 & Comunitaria & $17-33$ & Víctimas por encima de la media & Periodo de vida & Versión retrospectiva \\
\hline $\begin{array}{l}\text { Pereda, Abad y Guilera } \\
(2017)\end{array}$ & España & 101 & $\begin{array}{l}\text { Menores cumpliendo } \\
\text { medidas judiciales }\end{array}$ & $14-17$ & $\begin{array}{l}\text { Víctimas por encima de la media } \\
10 \% \text { superior }\end{array}$ & $\begin{array}{l}\text { Periodo de vida } \\
\text { Último año }\end{array}$ & Adaptación española \\
\hline $\begin{array}{l}\text { Pinto-Cortez, Pereda y } \\
\text { Álvarez-Lister (2017) }\end{array}$ & Chile & 706 & Comunitaria & $12-17$ & $\begin{array}{l}\text { Víctimas por encima de la media } \\
10 \% \text { superior }\end{array}$ & $\begin{array}{l}\text { Periodo de vida } \\
\text { Último año }\end{array}$ & $\begin{array}{l}\begin{array}{l}\text { Adaptación } \\
\text { española } \\
\text { chileno }\end{array} \\
\end{array}$ \\
\hline $\begin{array}{l}\text { Sterzing, Ratliff, Gartner, } \\
\text { McGeough y Johnson } \\
(2017)\end{array}$ & $\begin{array}{l}\text { Estados } \\
\text { Unidos }\end{array}$ & 1.177 & Comunitaria & $14-19$ & Víctimas por encima de la media & Último año & $\begin{array}{l}\text { Versión abreviada } \\
\text { modificada por los autores }\end{array}$ \\
\hline $\begin{array}{l}\text { Turner, Shattuck, } \\
\text { Finkelhor y Hamby (2016) }\end{array}$ & $\begin{array}{l}\text { Estados } \\
\text { Unidos }\end{array}$ & 2.312 & Comunitaria & $10-17$ & Análisis de clase latente & Último año & $\begin{array}{l}\text { Versión específica } \\
\text { realizada para la Segunda } \\
\text { Encuesta Nacional de la } \\
\text { Exposición de los niños a } \\
\text { la Violencia (NatSCEV II) }\end{array}$ \\
\hline
\end{tabular}

Revista Española de Investigación Criminológica

Artículo 6, Número 18 (2020)

https://doi.org/10.46381/reic.v18i1.399

www.criminologia.net

ISSN: 1696-9219 


\begin{tabular}{|c|c|c|c|c|c|c|c|}
\hline Autores y año & País & $\mathbf{N}$ & Tipo de muestra & Edad & Método & Periodo & Versión del cuestionario \\
\hline $\begin{array}{l}\text { Lätsch, Nett y Hümbelin } \\
\text { (2016) }\end{array}$ & Suiza & 6.749 & Comunitaria & $14-17$ & Víctimas por encima de la media & Periodo de vida & Versión original \\
\hline $\begin{array}{l}\text { Aho, Gren-Landell y } \\
\text { Svedin }(2016)\end{array}$ & Suecia & 5.960 & Comunitaria & 17 & $10 \%$ superior & Periodo de vida & $\begin{array}{l}\text { Versión original reducida } \\
\text { por los autores de este } \\
\text { estudio }\end{array}$ \\
\hline $\begin{array}{l}\text { Guerra, Pereda, Guilera y } \\
\text { Abad (2016) }\end{array}$ & España & 144 & Clínica & $12-17$ & Víctimas por encima de la media & Periodo de vida & Adaptación española \\
\hline $\begin{array}{l}\text { Kretschmar, Tossone, } \\
\text { Butcher y Flannery (2016) }\end{array}$ & $\begin{array}{l}\text { Estados } \\
\text { Unidos }\end{array}$ & 467 & $\begin{array}{l}\text { Programas de atención a } \\
\text { la infancia (justicia } \\
\text { juvenil, bienestar infantil } \\
\text { y salud mental) }\end{array}$ & $8-18$ & Análisis de clase latente & Último año & $\begin{array}{l}\text { Versión abreviada } \\
\text { ampliada por los autores de } \\
\text { este estudio }\end{array}$ \\
\hline $\begin{array}{l}\text { Pereda, Abad y Guilera } \\
(2015)\end{array}$ & España & 149 & Clínica & $12-17$ & $\begin{array}{l}\text { Víctimas por encima de la media } \\
10 \% \text { superior }\end{array}$ & $\begin{array}{l}\text { Periodo de vida } \\
\text { Último año }\end{array}$ & Adaptación española \\
\hline $\begin{array}{l}\text { Segura, Pereda, Abad y } \\
\text { Guilera (2015) }\end{array}$ & España & 129 & Acogimiento residencial & $12-17$ & $\begin{array}{l}\text { Víctimas por encima de la media } \\
10 \% \text { superior }\end{array}$ & $\begin{array}{l}\text { Periodo de vida } \\
\text { Último año }\end{array}$ & Adaptación española \\
\hline $\begin{array}{l}\text { Pinto-Cortez y Venegas } \\
(2015)\end{array}$ & Chile & 706 & Comunitaria & $12-17$ & Víctimas por encima de la media & Periodo de vida & $\begin{array}{l}\text { Adaptación versión } \\
\text { española al lenguaje local }\end{array}$ \\
\hline $\begin{array}{l}\text { Bogolyubova, Skochilov y } \\
\text { Smykalo (2015) }\end{array}$ & Rusia & 743 & Estudiantes universitarios & $19-25$ & $\begin{array}{l}\text { Víctimas por encima de la media } \\
10 \% \text { superior }\end{array}$ & Periodo de vida & Versión retrospectiva \\
\hline $\begin{array}{l}\text { Le, Holton, Nguyen, Wolfe } \\
\text { y Fisher (2015) }\end{array}$ & Vietnam & 1.606 & Comunitaria & $15-17$ & Víctimas por encima de la media & Periodo de vida & $\begin{array}{l}\text { Versión original ampliada } \\
\text { por los autores de este } \\
\text { estudio }\end{array}$ \\
\hline $\begin{array}{l}\text { Pereda, Guilera y Abad } \\
\text { (2014) }\end{array}$ & España & 1.107 & Comunitaria & $12-17$ & $\begin{array}{l}\text { Víctimas por encima de la media } \\
10 \% \text { superior }\end{array}$ & $\begin{array}{l}\text { Periodo de vida } \\
\text { Último año }\end{array}$ & Adaptación española \\
\hline $\begin{array}{l}\text { Cyr, Clément } \\
\text { Chamberland (2014) }\end{array}$ & Canadá & 1.401 & Comunitaria & $2-11$ & Víctimas por encima de la media & Periodo de vida & $\begin{array}{l}\text { Versión para cuidadores } \\
\text { principales }\end{array}$ \\
\hline $\begin{array}{l}\text { Álvarez-Lister, Pereda, } \\
\text { Abad, Guilera y GReVIA } \\
\text { (2014) }\end{array}$ & España & 132 & Clínica & $12-17$ & $\begin{array}{l}\text { Análisis de conglomerados } \\
\text { tradicional }\end{array}$ & Periodo de vida & Adaptación española \\
\hline Cyr et al. (2013) & Canadá & 2.801 & Comunitaria & $2-17$ & $\begin{array}{l}\text { Víctimas por encima de la media } \\
10 \% \text { superior }\end{array}$ & $\begin{array}{l}\text { Periodo de vida } \\
\text { Último año }\end{array}$ & $\begin{array}{l}\text { Versión para cuidadores } \\
\text { principales }\end{array}$ \\
\hline
\end{tabular}

Artículo 6, Número 18 (2020)

https://doi.org/10.46381/reic.v18i1.399

www.criminologia.net

ISSN: 1696-9219 


\begin{tabular}{|c|c|c|c|c|c|c|c|}
\hline Autores y año & País & $\mathbf{N}$ & Tipo de muestra & Edad & Método & Periodo & Versión del cuestionario \\
\hline Chan (2013) & China & 18.341 & Comunitaria & $15-17$ & Víctimas por encima de la media & $\begin{array}{l}\text { Periodo de vida } \\
\text { Último año }\end{array}$ & Versión china \\
\hline $\begin{array}{l}\text { Dong, Cao, Cheng, Cui y } \\
\operatorname{Li}(2013)\end{array}$ & China & 3.155 & Comunitaria & $12-18$ & Víctimas por encima de la media & Último año & $\begin{array}{l}\text { Versión china modificada } \\
\text { por los autores de este } \\
\text { estudio }\end{array}$ \\
\hline Cyr et al. (2012) & Canadá & 220 & $\begin{array}{l}\text { Menores en el servicio de } \\
\text { protección infantil }\end{array}$ & $2-17$ & Víctimas por encima de la media & Último año & $\begin{array}{l}\text { Versión original para } \\
\text { menores } \\
\text { Versión para cuidadores } \\
\text { principales }\end{array}$ \\
\hline $\begin{array}{l}\text { Soler, Paretilla, Kirchner y } \\
\text { Forns (2012) }\end{array}$ & España & 722 & Comunitaria & $14-18$ & $10 \%$ superior & Último año & Versión original \\
\hline $\begin{array}{l}\text { Finkelhor, Shattuck, } \\
\text { Turner, Ormrod y Hamby } \\
\text { (2011) }\end{array}$ & $\begin{array}{l}\text { Estados } \\
\text { Unidos }\end{array}$ & 4.046 & Comunitaria & $2-17$ & $10 \%$ superior & Último año & $\begin{array}{l}\text { Versión específica del } \\
\text { original mejorada y } \\
\text { ampliada para la Encuesta } \\
\text { Nacional de la Exposición } \\
\text { de los niños a la Violencia } \\
\text { (NatSCEV) }\end{array}$ \\
\hline $\begin{array}{l}\text { Turner, Finkelhor y } \\
\text { Ormrod (2010) }\end{array}$ & $\begin{array}{l}\text { Estados } \\
\text { Unidos }\end{array}$ & 4.053 & Comunitaria & $2-17$ & $10 \%$ superior & Periodo de vida & $\begin{array}{l}\text { Versión original ampliada } \\
\text { para menores y para } \\
\text { cuidadores principales }\end{array}$ \\
\hline
\end{tabular}

Revista Española de Investigación Criminológica

Artículo 6, Número 18 (2020)

https://doi.org/10.46381/reic.v18i1.399

Www.criminologia.net

ISSN: 1696-9219 
En Canadá las cifras varían según el estudio entre un 22\% (Cyr, Clément y Chamberland, 2014) y un 38\% (Cyr et al., 2017). Las cifras más elevadas se encuentran en Rusia, con un 70.1\% (Bogolyubova, Skochilov y Smykalo, 2015), y en España y Chile. En España, Guerra, Pereda, Guilera y Abad (2016) encontraron porcentajes del 50.1\% de polivíctimas durante el periodo de vida, estando el $34.7 \%$ en el grupo de la denominada polivictimización de nivel bajo y el $15.4 \%$ en la polivictimización de nivel alto. En Chile la cifra más baja es un 43.5\% (Pinto-Cortez y Venegas, 2015), llegándose a alcanzar un 83.9\%, de los cuales el 23.3\% fueron polivíctimas de nivel bajo y el 60.6\% de nivel alto (Guerra, Inostroza, Villegas, Villalobos y Pinto-Cortez, 2017).

Esta panorámica debe interpretarse con cautela. Uno de los motivos es que los puntos de corte que determinan si se es víctima o polivíctima varían en cada estudio (Guerra et al., 2017). Para ejemplificar, fijémonos en el 50.1\% de polivictimización durante el periodo de vida hallado en España (Guerra, Pereda, Guilera y Abad, 2016) y el 83.9\% en Chile (Guerra et al., 2017).

En el primer caso, las autoras establecen los puntos de corte basándose en la media de victimizaciones sufridas por los menores de su muestra. De este modo, las polivíctimas de nivel bajo son aquellas que han sufrido de 6 a 10 tipos de victimizaciones, mientras que las polivíctimas de nivel alto son las que han sufrido de 11 a 18 tipos.

En el segundo caso, los puntos de corte son los mismos que propusieron Finkelhor et al. (2005). Por tanto, las polivíctimas son aquellas que han sufrido 4 o más tipos de victimizaciones, siendo polivíctimas de nivel bajo aquellas que han sufrido de 4 a 6 tipos, y polivíctimas de nivel alto las que han sufrido 7 o más (Tabla 2). Aunque no existe ningún puntaje que determine la presencia o ausencia de polivictimización, éstos suelen utilizarse con frecuencia (Guerra et al., 2017). 
Tabla 2.

Comparación de los puntos de corte entre los estudios que utilizan el método 'víctimas por encima de la media' para analizar la polivictimización durante el periodo de vida

\begin{tabular}{|c|c|c|c|c|}
\hline Estudio & País & Puntos de corte & $\begin{array}{l}\text { Prevalencia } \\
\text { por niveles }\end{array}$ & $\begin{array}{l}\text { Prevalencia } \\
\text { total }\end{array}$ \\
\hline $\begin{array}{l}\text { Segura et al. } \\
(2018)\end{array}$ & España & $\begin{array}{l}\begin{array}{l}\text { Polivictimización } \\
\text { victimizaciones })\end{array}\end{array}$ & - & $27 \%^{a}$ \\
\hline $\begin{array}{l}\text { Guerra et al. } \\
(2016)\end{array}$ & España & $\begin{array}{l}\text { Polivictimización de nivel bajo ( } 6 \text { a } 10 \\
\text { victimizaciones) } \\
\text { Polivictimización de nivel alto ( } 11 \text { a } 18 \\
\text { victimizaciones) }\end{array}$ & $\begin{array}{l}34.7 \% \\
15.4 \%\end{array}$ & $50.1 \%$ \\
\hline $\begin{array}{l}\text { Guerra et al. } \\
(2017)\end{array}$ & Chile & $\begin{array}{l}\text { Polivictimización de nivel bajo ( } 4 \text { a } 6 \\
\text { victimizaciones) } \\
\text { Polivicitmización nivel alto ( } 7 \text { o más } \\
\text { victimizaciones) }\end{array}$ & $\begin{array}{l}23.3 \% \\
60.6 \%\end{array}$ & $83.9 \% \%^{\mathrm{a}}$ \\
\hline Chan (2013) & China & $\begin{array}{l}\begin{array}{l}\text { Polivictimización } \\
\text { victimizaciones })\end{array}\end{array} \quad \begin{array}{lll}4 & \text { o } & \text { más }\end{array}$ & - & $14 \%^{\mathrm{a}}$ \\
\hline Cyr et al. (2017) & Canadá & $\begin{array}{l}\begin{array}{l}\text { Polivictimización } \\
\text { victimizaciones }\end{array}\end{array} \quad$ ( 4 o $\quad$ más & - & $38 \% \%^{a, c}$ \\
\hline $\begin{array}{l}\text { Pinto-Cortez y } \\
\text { Venegas (2015) }\end{array}$ & Chile & $\begin{array}{l}\text { Polivictimización de nivel bajo ( } 7 \text { o } \\
\text { más victimizaciones) } \\
\text { Polivictimización de nivel alto ( } 11 \text { o } \\
\text { más victimizaciones) }\end{array}$ & $\begin{array}{l}30.3 \% \\
13.2 \%\end{array}$ & $45.3 \%$ \\
\hline Cyr et al. (2014) & Canadá & $\begin{array}{l}\begin{array}{l}\text { Polivictimización } \\
\text { victimizaciones }\end{array}\end{array} \quad$ ( 4 o $\quad$ más & - & $22 \%{ }^{b, d}$ \\
\hline Le et al. (2015) & Vietnam & $\begin{array}{l}\text { Polivictimización nivel bajo ( } 11 \text { a } 14 \\
\text { victimizaciones) } \\
\text { Polivictimización nivel alto ( } 15 \text { o más } \\
\text { victimizaciones) }\end{array}$ & $\begin{array}{l}17.2 \% \\
14.6 \%\end{array}$ & $31.8 \%$ \\
\hline $\begin{array}{l}\text { Bogolyubova et } \\
\text { al. }(2015)\end{array}$ & Rusia & 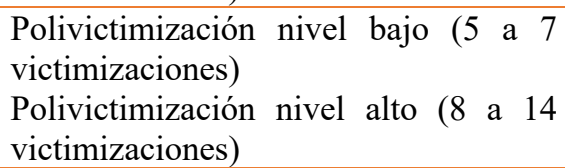 & $\begin{array}{l}24.66 \% \\
45.45 \%\end{array}$ & $70.1 \%{ }^{\mathrm{a}}$ \\
\hline $\begin{array}{l}\text { Lästch et al. } \\
\text { (2016) }\end{array}$ & Suiza & $\begin{array}{l}\begin{array}{l}\text { Polivictimización } \\
\text { victimizaciones) }\end{array}\end{array}$ & - & $22 \%^{\mathrm{a}}$ \\
\hline
\end{tabular}

${ }^{a}$ Según los criterios de Finkelhor, et al. (2005)

${ }^{\mathrm{b}}$ Según los criterios de Finkelhor, Ormrod y Turner (2007b)

${ }^{\mathrm{c}}$ El $18 \%$ del grupo de mayor edad (16 a 17 años) reportó más de 7

${ }^{\mathrm{d}}$ En el grupo de mayor edad (6 a 11 años)

El segundo método de análisis es el propuesto por Finkelhor, Ormrod y Turner (2009), con el cual se consideran polivíctimas a aquellas que se encuentran dentro del 10\% superior más victimizado. Este método pretende detectar la polivictimización extrema y es utilizado, principalmente, para analizar la victimización durante el periodo de vida de niños, 
niñas y adolescentes. Para ello, el método identifica un punto de corte que se corresponde con el número más alto de victimizaciones sufrido por el $90 \%$ de la muestra.

En España las tasas de polivictimización halladas mediante este método rondan entre un 7.9\% (Pereda, Guilera y Abad, 2014) y un 53.1\% (Segura et al., 2015). En Chile varían de un $8.6 \%$ (Pinto-Cortez, Flores- Jara, Pereda y Guerra, 2019) a un 14.7\% (Pinto-Cortez, Guitérrez-Echegoyen y Henríquez, 2018), mientras que en Rusia y Suecia son, respectivamente un 10.8\% (Bogolyubova et al., 2015) y un 10.3\% (Aho, Gren-Landell y Göran, 2016).

Finkelhor et al. (2009) determinaron que las experiencias de victimización se van acumulando con la edad. A mayor edad, mayor tiempo de exposición, y por consiguiente, mayores probabilidades de victimización. En Estados Unidos se llevó a cabo un estudio que identificó a las polivíctimas como aquellas que habían sufrido 11 o más tipos de victimizaciones durante su periodo de vida. Los resultados mostraron que había un 5.4\% de polivíctimas en el grupo de edad de 2 a 5 años, un $12.6 \%$ en el grupo de 6 a 9 años, un $21.2 \%$ en el de 10 a 13 años y, por último, un $60.8 \%$ en el de 14 a 17 años (Turner, Finkelhor y Ormrod, 2010). Se puede apreciar un progresivo aumento de la polivictimización conforme se incrementa la edad. No obstante, el hecho de establecer los mismos puntos de corte en todas las edades podría llevar a una errónea identificación de las polivíctimas en los distintos grupos de edad, pudiendo ser los puntos de corte demasiado extremos para las polivíctimas de menor edad (Finkelhor et al., 2009).

Por este motivo, se considera más adecuado establecer puntos de corte para cada grupo de edad. De este modo, Pereda, Guilera y Abad (2014) hallaron en el rango de edad de 12 a 14 años un punto de corte de 7 o más tipos de victimizaciones y de 9 o más para el rango de 15 a 17 años. Igualmente, Segura, Pereda, Abad y Guilera (2015) para los mismos rangos de edad hallaron puntos de corte de 13 o más y de 17 o más respectivamente. Efectivamente, en estos casos los niños de mayor edad son aquellos que muestran un mayor número de victimizaciones; sin embargo, los datos muestran que no ocurre siempre así. En Chile, se encontró que eran los adolescentes de menor edad los que mostraban mayor número de victimizaciones, habiendo sufrido 14 o más tipos aquellos con edades comprendidas entre

Revista Española de Investigación Criminológica

Artículo 6, Número 18 (2020)

https://doi.org/10.46381/reic.v18i1.399

www.criminologia.net

ISSN: 1696-9219 
los 12 y los 14 años, y 12 o más tipos en las edades de 15 a 17 años (Pinto-Cortez et al., 2017) (Tabla 4).

El método del 10\% superior más victimizado es considerado por Finkelhor et al. (2009) el más adecuado para medir la victimización durante el periodo de vida; no obstante, diferentes estudios también analizan el último año de vida. Estos estudios han mostrado que el porcentaje de menores que se encuentran dentro del percentil 10 se sitúan entre un 5.1\% (Soler, Paretilla, Kirchner y Forns, 2012), un 11.9\% (Pinto-Cortez et al., 2017) y un 41.6\% (Pereda et al., 2017). Teniendo en cuenta la acumulación de experiencias de victimización con el incremento de la edad, para este marco temporal también se establecen diferentes puntos de corte por grupos de edad, como realizan Finkelhor, Shattuck, Turner, Ormrod y Hamby (2011), siendo de 5 o más en el grupo de 2 a 5 años, y aumentando progresivamente hasta llegar a 8 o más en el grupo de 14 a 17 años (Tabla 3).

\section{Tabla 3.}

Estudios que utilizan el 10\% superior más victimizado con los puntos de corte y las prevalencias en los dos marcos temporales

\begin{tabular}{|c|c|c|c|c|}
\hline Estudio & Lugar & Periodo de tiempo & Puntos de corte & Prevalencia \\
\hline \multirow{2}{*}{$\begin{array}{l}\text { Segura et al. } \\
\text { (2018) }\end{array}$} & \multirow{2}{*}{ España } & Periodo de vida & $8+$ & $8.1 \%$ \\
\hline & & Último año de vida & $6+$ & $7 \%$ \\
\hline \multirow{3}{*}{$\begin{array}{l}\text { Pereda et al. } \\
\text { (2017) }\end{array}$} & \multirow[t]{3}{*}{ España } & Periodo de vida & $9+$ & $65.3 \%^{\mathrm{a}}$ \\
\hline & & \multirow[t]{2}{*}{ Último año de vida } & $6+$ & $41.6 \%{ }^{\mathrm{a}}$ \\
\hline & & & $10+$ & $7.9 \%$ \\
\hline \multirow{2}{*}{$\begin{array}{l}\text { Indias-García y } \\
\text { De Paúl- } \\
\text { Ochotorena } \\
(2017)\end{array}$} & \multirow[t]{2}{*}{ España } & \multirow[t]{2}{*}{ Periodo de vida } & $11+$ & $13,5 \%$ \\
\hline & & & $8+$ & $28.1 \%^{\mathrm{a}}$ \\
\hline \multirow[t]{4}{*}{$\begin{array}{l}\text { Segura et al. } \\
(2015)\end{array}$} & \multirow[t]{4}{*}{ España } & \multirow[t]{2}{*}{ Periodo de vida } & $\begin{array}{l}12 \text { a } 14 \text { años: } 13+ \\
15 \text { a } 17 \text { años: } 17+ \\
\text { Toda la muestra: } 15+\end{array}$ & $\begin{array}{l}12.6 \% \\
10.5 \% \\
14.3 \%\end{array}$ \\
\hline & & & $\begin{array}{l}12 \text { a } 14 \text { años: } 7+ \\
15 \text { a } 17 \text { años: } 9+ \\
\text { Toda la muestra: } 8+\end{array}$ & $\begin{array}{l}50.2 \%^{\mathrm{a}} \\
59.9 \%^{\mathrm{a}} \\
53.1 \%^{\mathrm{a}}\end{array}$ \\
\hline & & \multirow[t]{2}{*}{ Último año de vida } & $\begin{array}{l}12 \text { a } 14 \text { años: } 7+ \\
15 \text { a } 17 \text { años: } 8+ \\
\text { Toda la muestra: } 8+\end{array}$ & $\begin{array}{l}11 \% \\
12.2 \% \\
10.2 \%\end{array}$ \\
\hline & & & $\begin{array}{l}12 \text { a } 14 \text { años: } 6+ \\
15 \text { a } 17 \text { años: } 6+ \\
\text { Toda la muestra: } 6+\end{array}$ & $\begin{array}{l}18.8 \%^{\mathrm{a}} \\
33.7 \%^{\mathrm{a}} \\
26.5 \%^{\mathrm{a}}\end{array}$ \\
\hline
\end{tabular}

Revista Española de Investigación Criminológica

Artículo 6, Número 18 (2020)

https://doi.org/10.46381/reic.v18i1.399

www.criminologia.net

ISSN: 1696-9219 


\begin{tabular}{|c|c|c|c|c|}
\hline Estudio & Lugar & Periodo de tiempo & Puntos de corte & Prevalencia \\
\hline \multirow{2}{*}{$\begin{array}{l}\text { Pereda et al. } \\
\text { (2015) }\end{array}$} & \multirow[t]{2}{*}{ España } & Periodo de vida & $9+$ & $32.2 \%^{\mathrm{a}}$ \\
\hline & & Último año de vida & $6+$ & $20.1 \%^{\mathrm{a}}$ \\
\hline \multirow[t]{2}{*}{$\begin{array}{l}\text { Pereda et al. } \\
\text { (2014) }\end{array}$} & \multirow[t]{2}{*}{ España } & Periodo de vida & $\begin{array}{l}12 \text { a } 14 \text { años: } 7+ \\
15 \text { a } 17 \text { años: } 9+ \\
\text { Toda la muestra: } 8+\end{array}$ & $\begin{array}{l}9.8 \% \\
7.6 \% \\
7.9 \%\end{array}$ \\
\hline & & Último año de vida & $\begin{array}{l}12 \text { a } 14 \text { años: } 6^{+} \\
15 \text { a } 17 \text { años: } 6^{+} \\
\text {Toda la muestra: } 6^{+}\end{array}$ & $\begin{array}{l}6.5 \% \\
7.4 \% \\
7 \%\end{array}$ \\
\hline $\begin{array}{l}\text { Soler et al. } \\
\text { (2012) }\end{array}$ & España & Último año de vida & $9+$ & $5.1 \% \mathrm{o}^{\mathrm{b}}$ \\
\hline $\begin{array}{l}\text { Pinto-Cortez et } \\
\text { al. (2019) }\end{array}$ & Chile & Periodo de vida & $\begin{array}{l}11+ \\
12+\end{array}$ & $\begin{array}{l}13 \%^{\mathrm{c}} \\
8.6 \%^{\mathrm{c}}\end{array}$ \\
\hline $\begin{array}{l}\text { Pinto-Cortez et } \\
\text { al. }(2018)\end{array}$ & Chile & Periodo de vida & $18+$ & $14.7 \%$ \\
\hline \multirow[t]{2}{*}{$\begin{array}{l}\text { Pinto-Cortez et } \\
\text { al. (2017) }\end{array}$} & \multirow[t]{2}{*}{ Chile } & Periodo de vida & $\begin{array}{l}12 \text { a } 14 \text { años: } 14+ \\
15 \text { a } 17 \text { años: } 12+ \\
\text { Toda la muestra: } 12+\end{array}$ & $\begin{array}{l}10 \% \\
10.1 \% \\
10.5 \%\end{array}$ \\
\hline & & Último año de vida & $\begin{array}{l}12 \text { a } 14 \text { años: } 11+ \\
15 \text { a } 17 \text { años: } 8+ \\
\text { Toda la muestra: } 8+\end{array}$ & $\begin{array}{l}10 \% \\
11.4 \% \\
11.9 \%\end{array}$ \\
\hline $\begin{array}{l}\text { Finkelhor et al. } \\
\text { (2011) }\end{array}$ & EEUU & Último año de vida & $\begin{array}{l}2 \text { a } 5 \text { años: } 5+ \\
6 \text { a } 9 \text { años: } 6+ \\
10 \text { a } 13 \text { años: } 7+ \\
14 \text { a } 17 \text { años: } 8+\end{array}$ & $\begin{array}{l}21 \% \\
16 \% \\
19 \% \\
19 \% \\
\end{array}$ \\
\hline $\begin{array}{l}\text { Turner et al. } \\
(2010)\end{array}$ & EEUU & Periodo de vida & $\begin{array}{l}2 \text { a } 5 \text { años: } 11+ \\
6 \text { a } 9 \text { años: } 11+ \\
10 \text { a } 13 \text { años: } 11+ \\
14 \text { a } 17 \text { años: } 11+\end{array}$ & $\begin{array}{l}5.4 \% \\
12.6 \% \\
21.2 \% \\
60.8 \% \\
\end{array}$ \\
\hline $\begin{array}{l}\text { Bogolyubova et } \\
\text { al. }(2015)\end{array}$ & Rusia & Periodo de vida & $15+$ & $10.8 \%{ }^{\mathrm{d}}$ \\
\hline $\begin{array}{l}\text { Aho et al. } \\
(2016)\end{array}$ & Suecia & Periodo de vida & $10+$ & $10.3 \%$ \\
\hline
\end{tabular}

${ }^{a}$ Según los criterios de Pereda, Guilera y Abad (2014)

${ }^{\mathrm{b}} \mathrm{Se}$ corresponde con aquellos que han sufrido 11 o más tipos de victimización

${ }^{\mathrm{c}}$ Comparación entre una muestra de menores en minoría étnica (11+) y no minoría étnica $(12+)$

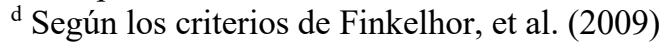

El tercer método utilizado para detectar la polivictimización hace referencia a las técnicas de agrupamiento, cuya finalidad es identificar los perfiles de victimización de aquellas personas con elevadas medias de exposición a diferentes tipos de victimización, es decir, las polivíctimas (Segura et al., 2018). Dentro de las técnicas de agrupamiento se usan dos enfoque metodológicos; por un lado, el análisis por conglomerados tradicional, como es el caso del estudio de Álvarez-Lister, Pereda, Abad, Guilera y GReVIA (2014), donde se

Artículo 6, Número 18 (2020)

https://doi.org/10.46381/reic.v18i1.399

www.criminologia.net

ISSN: 1696-9219 
halló un $12.9 \%$ de adolescentes que habían sufrido polivictimización durante su infancia y adolescencia; por otro lado, el análisis de clase latente, hallando porcentajes de polivictimización en el periodo de vida del 17.8\% (Turner, Shattuck, Finkelhor y Hamby, 2016) y del 18.3\% (Segura et al., 2018), y durante el último año de vida del 6.2\% (Segura et al., 2018) y del 23.1\% (Kretchsmar, Tossone, Butcher y Flannery, 2016).

Estos enfoques permiten tener en cuenta una multiplicidad de factores que podrían estar definiendo los perfiles de victimización más allá de focalizarse en el número de victimizaciones sufridas por cada individuo. Así, agrupan y clasifican a aquellos individuos que presentan un mismo perfil de victimización, detectando también un subgrupo de individuos con una alta exposición a múltiples tipos de victimización.

De los tres métodos, el análisis de clase latente ha sido el menos utilizado hasta la fecha. Curiosamente, los resultados del estudio de Segura, Pereda y Guilera (2018) muestran que este método tiene un alto grado de consistencia con el 10\% superior más victimizado cuando se analiza el último año de vida, y con el método de víctimas por encima de la media cuando se analiza el periodo de vida.

Del mismo modo, las autoras determinaron que el método de víctimas por encima de la media y el $10 \%$ superior más victimizado tenían el menor grado de consistencia. Sin embargo, estos dos métodos han sido aplicados simultáneamente en una gran cantidad de estudios (Bogolyubova et al., 2015; Pereda et al., 2017; Pereda, Abad y Guilera, 2015; Pereda et al., 2014; Pinto-Cortez et al., 2018; Pinto-Cortez et al., 2017; Segura et al., 2015).

En cuanto a los criterios de cada estudio para establecer los puntos de corte, algunas investigaciones sirven como marco de referencia para estudios posteriores. En muchas ocasiones se toman como referencia los criterios de Finkelhor et al. (2005) y de Finkelhor et al. (2009) (Tabla 2 y Tabla 3). Conviene matizar que, debido al modo en que se contabilizan las victimizaciones, Finkelhor et al. (2005) establecen diferentes puntos de corte para la clasificación de los grupos de víctimas, polivíctimas de nivel bajo y polivíctimas de nivel alto. Cuestión que abordaremos posteriormente. 
En España, el estudio de Pereda et al. (2014) es el marco de referencia para investigaciones como las de Indias-García y De Paúl-Ochotorena (2017), Pereda, Abad y Guilera (2015, 2017), Segura et al. (2015), entre otras.

Por último, el tipo de muestra también determina el establecimiento de unos u otros criterios. Así, Pinto-Cortez, Gutiérrez-Echegoyen y Henríquez (2018) siguen los criterios de Bogolyubova, Skochilov y Smykalo (2015) al analizar muestras de universitarios mediante una versión retrospectiva del Cuestionario de Victimización Juvenil.

\subsection{Características de la muestra}

La investigación en polivictimización, ha mostrado que, debido a sus características específicas, ciertos grupos de niños, niñas y adolescentes son más vulnerables frente a la victimización, como son aquellos menores con un diagnóstico clínico de salud mental. Un ejemplo lo encontramos en el estudio desarrollado por Álvarez-Lister, Pereda, Guilera, Abad y Segura (2017), donde comparan un grupo de pacientes ambulatorios en edad adolescente con un grupo de escolares, siendo las tasas de polivictimización del $40.6 \%$ y del 13.8\% respectivamente durante el último año de vida. También se han hallado en muestras clínicas similares porcentajes del 40.3\% (Pereda et al., 2015) en el último año de vida, y del 50.1\% (Guerra et al., 2016) y del 32.2\% (Pereda et al., 2015) durante el periodo de vida. El porcentaje más bajo hallado ha sido de un $12.9 \%$, con una media de victimizaciones de 13.65 (SD = 2.34, Álvarez-Lister, Pereda, Abad, Guilera y GReVIA, 2014).

La polivictimización tiene un efecto negativo en los procesos de integración y adaptación social, y está asociada con problemas emocionales y conductuales (Finkelhor, Ormrod y Turner, 2007). La investigación muestra que aquellos adolescentes inmersos en los servicios de protección a la infancia (Cyr et al., 2012; Segura et al., 2015) y aquellos que están involucrados en el sistema de justicia juvenil (Pereda et al., 2017) también muestran mayores niveles de polivictimización durante su infancia.

En Canadá se encontraron cifras de polivictimización durante el último año de vida de un 8\% (Cyr et al., 2013) y un 54\% (Cyr et al., 2012), correspondiendo el primer dato a

\section{Revista Española de Investigación Criminológica}

Artículo 6, Número 18 (2020)

https://doi.org/10.46381/reic.v18i1.399

www.criminologia.net

ISSN: 1696-9219 
una muestra comunitaria y el segundo a una de menores que se encuentran en el servicio de protección infantil. Del mismo modo, en España se halló que un 45,2\% de una muestra de menores que estaban en acogimiento residencial habían sufrido polivictimización durante su último año de vida (Segura et al., 2015), hallándose un porcentaje de un 20\% en una muestra comunitaria (Pereda et al., 2014).

Teniendo en cuenta los aspectos metodológicos de los que hemos hablado hasta ahora, estos estudios están sujetos a comparación porque (a) utilizan el mismo método de análisis, (b) establecen los mismos puntos de corte, (c) analizan el mismo periodo de tiempo y (d) las edades de las muestras se corresponden entre sí (de 2 a 17 años en Canadá y de 12 a 17 años en los estudios españoles) (Tabla 4).

\section{Tabla 4.}

Comparación entre prevalencias de polivictimización atendiendo al tipo de muestra, el método utilizado, los puntos de corte establecidos y el periodo de tiempo analizado

\begin{tabular}{|l|l|l|l|l|l|}
\hline Estudio & Muestra & $\begin{array}{l}\text { Periodo de } \\
\text { tiempo }\end{array}$ & Método & $\begin{array}{l}\text { Punto de } \\
\text { corte }\end{array}$ & Prevalencia \\
\hline $\begin{array}{l}\text { Cyr et al. } \\
(2013)\end{array}$ & Comunitaria & $\begin{array}{l}\text { Último año de } \\
\text { vida }\end{array}$ & $\begin{array}{l}\text { Víctimas por } \\
\text { encima de la } \\
\text { media }\end{array}$ & $4+$ & $8 \%$ \\
\hline $\begin{array}{l}\text { Cyr et al. } \\
(2012)\end{array}$ & $\begin{array}{l}\text { Sistema de } \\
\text { protección } \\
\text { infantil }\end{array}$ & $\begin{array}{l}\text { Último año de } \\
\text { vida }\end{array}$ & $\begin{array}{l}\text { Víctimas por } \\
\text { encima de la } \\
\text { media }\end{array}$ & $4+$ & $54 \%$ \\
\hline $\begin{array}{l}\text { Pereda et al. } \\
(2014)\end{array}$ & Comunitaria & $\begin{array}{l}\text { Último año de } \\
\text { vida }\end{array}$ & $\begin{array}{l}\text { Víctimas por } \\
\text { encima de la } \\
\text { media }\end{array}$ & $4+$ & $20 \%$ \\
\hline $\begin{array}{l}\text { Segura et al. } \\
(2015)\end{array}$ & $\begin{array}{l}\text { Sistema de } \\
\text { protección } \\
\text { infantil }\end{array}$ & $\begin{array}{l}\text { Último año de } \\
\text { vida }\end{array}$ & $\begin{array}{l}\text { Víctimas por } \\
\text { encima de la } \\
\text { media }\end{array}$ & $4+$ & $45.2 \%$ \\
\hline $\begin{array}{l}\text { Pereda et al. } \\
(2017)\end{array}$ & $\begin{array}{l}\text { Sistema de } \\
\text { justicia juvenil }\end{array}$ & $\begin{array}{l}\text { Último año de } \\
\text { vida }\end{array}$ & $\begin{array}{l}\text { Víctimas por } \\
\text { encima de la } \\
\text { media }\end{array}$ & $6+$ & $41.6 \%$ \\
\hline
\end{tabular}


Por otro lado, en una muestra de adolescentes que se hallaban cumpliendo medidas judiciales se encontró que un 41.6\% habían experimentado seis o más tipos de victimizaciones durante su último año de vida (Pereda et al., 2017) en comparación con el $20 \%$ de una muestra comunitaria (Pereda et al., 2014). En esta comparación conviene resaltar diversos aspectos:

Primero, las edades no coinciden, siendo de 14 a 17 años en la muestra de menores que están cumpliendo medidas judiciales y de 12 a 17 años en la muestra comunitaria. Quizás el hecho de que los adolescentes de mayor edad suelen acumular más experiencias que los de menor edad (Finkelhor et al., 2009), podría estar incrementando el número de polivíctimas en el primer caso.

Segundo, utilizando el mismo método de análisis (víctimas por encima de la media) los puntos de corte no coinciden, siendo 4+ para la muestra comunitaria y $6+$ para la muestra de menores que están cumpliendo medidas judiciales. Esto nos indica que los menores que están involucrados en el sistema de justicia juvenil sufren una media más elevada de victimizaciones. Cuando tomamos como referencia en la muestra comunitaria el método del $10 \%$, el punto de corte se iguala, siendo de $6+$. Podemos observar que con la misma media de victimizaciones se acentúa aún más la diferencia entre las prevalencias de polivictimización, ya que en este caso la cifra se reduce a un $7 \%$ en la muestra comunitaria (Tabla 4).

Por último, mediante un análisis de clase latente, Kretschmar, Tossone, Butcher y Flannery (2016) analizaron los patrones de polivictimización en un grupo de niños y adolescentes que se encontraban en diversos programas de atención infantil, servicios de bienestar infantil, justicia juvenil y salud mental. Los resultados mostraron un $23.1 \%$ de menores que habían sufrido altos niveles de exposición a la violencia durante el último año de vida. 


\subsection{Versión del Cuestionario de Victimización Juvenil}

Existen diversas versiones del Cuestionario de Victimización Juvenil (JVQ en adelante) y cada una incluye un número de ítems diferente, lo que significa que en cada caso se puede reportar un número total de victimizaciones distinto.

Los formatos en los que se pueden aplicar estar versiones son cuestionarios y entrevistas dirigidas a menores y a sus cuidadores principales. Así, dependiendo de la edad de la población que queramos estudiar se puede aplicar (Hamby et al., 2005):

- $\quad$ Entrevista para niños y niñas de 8 a 17 años.

- Entrevista para los cuidadores principales del menor (de 0 a 17 años) que hayan tenido un contacto regular con el niño o niña al menos durante el último año.

- Cuestionario autoadministrado (SAQ - Self Administered Questionnaire) para adolescentes de 12 a 17 años.

- Cuestionario autoadministrado para cuidadores principales (caregiver SAQ) cuyas preguntas se refieren a los niños de los 0 a los 17 años con los que han tenido, al menos durante el último año, un contacto regular.

- Entrevista narrativa, cuyo objetivo es obtener descripciones más detalladas de los eventos de victimización.

La versión original del JVQ se compone de 34 ítems que se corresponden con distintas formas de victimización, clasificadas en cinco módulos diferentes. El rango de victimizaciones posibles es de 0 a 34, siendo 34 el número máximo que un niño puede reportar. Varios estudios utilizan esta versión (p.e. Cyr et al., 2012, Cyr et al., 2017; Cyr et al., 2013; Lästch et al., 2012; Soler et al., 2012); sin embargo, posteriormente se incluyeron 2 ítems referentes a victimización electrónica en un sexto módulo, lo que amplía el rango de

victimizaciones hasta 36, siendo la versión más utilizada (p.e. Álvarez-Lister, Pereda, Guilera, Abad y Segura, 2017; Pereda et al, 2015, 2017; Pereda et al., 2014; Pinto-Cortez et al., 2019; Pinto-Cortez et al., 2017; Pinto-Cortez y Venegas, 2015; Segura et al., 2018). 
Existe también una versión abreviada que incluye 12 ítems. En este caso, el número máximo de victimizaciones que un niño o adolescente puede reportar es mucho menor que en los dos casos anteriores. Esta versión no tiene tanto poder predictivo para el grupo de polivíctimas (Finkelhor et al., 2005), sin embargo, esta versión es adecuada cuando no se dispone de tiempo suficiente para su administración. El estudio de Kretschmar et al. (2016) utiliza esta versión.

Diversas investigaciones utilizan la versión de los cuidadores principales (p.e. Cyr et al., 2013; Cyr et al., 2014), que incluye 32 formas de victimización al ser excluidos los ítems que hacen referencia a violencia en pareja y estupro. Esta versión permite conocer las experiencias de los más pequeños, pero debería considerarse la posibilidad de que los cuidadores principales desconozcan algunas victimizaciones o incluso que las escondan. Otros estudios (Bogolyubova et al., 2018; Guerra et al., 2017; Pinto-Cortez et al., 2018) utilizan la versión retrospectiva para adultos. El JVQ está diseñado para detectar la victimización sufrida en un periodo de tiempo relativamente corto, y aunque esta versión permita identificar la victimización completa durante la infancia y la adolescencia, se debe tener precaución puesto que algunos tipos específicos de victimización pueden ser difíciles de recordar con el paso del tiempo (Hamby et al., 2005).

Para poder aplicar el JVQ en los distintos países ha sido necesario realizar las traducciones y adaptaciones oportunas, lo que supone modificar también el número de ítems. Por ejemplo, en España el Grupo de Investigación en Victimización Infantil y Adolescente (GReVIA) de la Universidad de Barcelona llevó a cabo la adaptación, traducción y validación del instrumento (Pereda et al., 2018). Debido a que la definición legal del estupro no se corresponde con la de Estados Unidos, este ítem ( $\left.n^{\circ} 25\right)$ se ha eliminado del cuestionario (p.e. Álvarez-Lister, et al., 2014; Indias-García y De Paúl-Ochotorena, 2017; Pereda et al., 2014; Segura et al., 2015), ocurriendo lo mismo en Suecia (Aho et al., 2016). Otro ejemplo lo hallamos en China, donde también se realizó una adaptación al contexto del país, siendo eliminado el ítem referente a "Exposición a guerras o conflictos étnicos" al considerarlo innecesario alegando la ausencia de este tipo de violencia en el país (Dong, Cao, Cheng, Cui y Li, 2013).

Revista Española de Investigación Criminológica

Artículo 6, Número 18 (2020)

https://doi.org/10.46381/reic.v18i1.399

www.criminologia.net

ISSN: 1696-9219 
Por otro lado, las investigaciones también modifican los ítems atendiendo a las necesidades y objetivos del estudio. Sterzing, Ratliff, Gartner, McGeough y Johnson (2017), además de utilizar una versión abreviada, eliminan dos ítems: uno por no corresponderse con el periodo de tiempo que analizan y el otro porque se solapaba con un subtipo de victimización, el acoso escolar, que analizan en más profundidad con otro cuestionario. Otro de los estudios (Le et al., 2015) añade tres ítems referentes a violencia familiar, negligencia y acoso online. De este modo, el cuestionario se compuso de 37 ítems, partiendo de los 34 de la versión original. Lo mismo ocurre en el estudio de Kretschmar et al. (2016), quienes añaden a los 12 ítems de la versión abreviada otros tres, al considerarlos oportunos por las características específicas de la muestra con la que trabajan.

Otro ejemplo claro se puede observar en varias investigaciones de Estados Unidos. En el estudio de Finkelhor et al. (2011) se aplica una versión específica para la Encuesta Nacional de la Exposición de los niños a la Violencia (NatSCEV o National Survey of Children's Exposure to Violence) que partiendo de la original, es ampliada hasta una total de 48 ítems, y usando un rango de 0 a 36 para medir la polivictimización durante el último año de vida. Igualmente, otro estudio utiliza también una versión específica para la Segunda Encuesta Nacional de la Exposición de los niños a la Violencia (NatSCEV II) donde el total de ítems se amplía hasta los 51, siendo el rango de 0 a 25 para la polivictimización del último año de vida (Turner et al., 2016).

Además de la versión del instrumento, la manera de contabilizar los ítems también puede influir en la determinación de los puntos de corte, tal y como muestran Finkelhor et al. (2005), quienes propusieron dos maneras de puntaje.

Por un lado, como incidentes de victimización separados (SIV o Separate Incident Version), contándose únicamente las victimizaciones ocurridas en momentos y lugares diferentes, y estableciéndose un punto de corte de 4. El SIV ha sido utilizado por estudios como, Cyr et al. (2012) o Cyr et al. (2013). Por otro lado, como un recuento total de victimizaciones (SSV o Screener Sum Version), contándose aquellas que han ocurrido en el mismo lugar y momento, siendo en este caso el punto de corte de 5. Así se contabiliza en estudios como Lästch, Nett y Hümbelin (2016), Aho, Gren-Landell y Svedin (2016),

\section{Revista Española de Investigación Criminológica}


Bogolyubova et al. (2015), Indias-García y De Paúl-Ochotorena (2017) o Soler, Paretilla, Kirchner y Forns (2012). Al utilizar la versión abreviada, el punto de corte fue de 3.

Además, la manera de evaluar el instrumento se puede realizar a distintos niveles (Hamby et al., 2005; Hamby et al., 2011):

- A nivel de ítem, puntuando las respuestas afirmativas en cada ítem (p.e. Guerra et al., 2017; Indias-García y De Paúl-Ochotorena, 2017; Pinto-Cortez y Venegas, 2015) y como ítem re-puntuado, mediante preguntas de seguimiento para obtener información más completa sobre el incidente, por ejemplo, cuántas veces lo ha sufrido, quién fue el agresor, etc. (Cyr et al., 2012; Pereda et al., 2015; Pinto-Cortez et al., 2018; Turner et al., 2010).

- A nivel de módulo, considerando que se es víctima de un módulo determinado cuando se haya respondido afirmativamente al menos a uno de los ítems que lo componen (p.e. Álvarez-Lister et al., 2017; Indias-García y De Paúl-Ochotorena, 2017).

- Como categoría agregada, añadiendo a un tipo específico de victimización las diferentes maneras en la que se puede presentar (categoría de agresión física: abuso físico por parte de cuidadores, agresión física por parte de hermanos, agresión física por parte de compañeros, etc.) (p.e. Cyr et al., 2013; Cyr et al., 2017; Cyr et al., 2014; Finkelhor et al., 2011; Le et al., 2015; Turner et al., 2010).

Tal y como apuntan Sterzing et al. (2017) estos aspectos influyen en el establecimiento de los puntos de corte en cada estudio. El número de victimizaciones total que se pueden reportar, junto con la manera de contabilizar los ítems, pueden determinar que los puntos de corte sean más o menos elevados.

\section{Discusión}

El concepto de polivíctima está ampliamente reconocido, pero la ausencia de un acuerdo metodológico deriva en una multiplicidad de elementos que intervienen en la identificación de polivíctimas, y que da como resultado definiciones metodológicas diferentes. El presente

\section{Revista Española de Investigación Criminológica}


trabajo tiene como objetivo realizar una revisión del constructo de polivictimización desde una perspectiva metodológica, describiendo los elementos que deben considerarse en la interpretación de su prevalencia.

Hablar de la epidemiología de la polivictimización es complicado debido a esa multiplicidad de elementos que componen los criterios metodológicos de cada investigación. Utilizar los mismos criterios metodológicos permite hacer comparables los resultados obtenidos en diversos estudios, ya tengan muestras similares, como pueden ser las comunitarias, o muestras diferentes, por ejemplo, una comunitaria y una de riesgo. En este sentido, generalmente se adoptan como marcos de referencia para establecer los criterios metodológicos los estudios de Finkelhor et al. (2005) y de Finkelhor et al. (2009). Pero también otros estudios son utilizados como referencia, como es el caso de Pereda et al. (2014) en el contexto español.

Adoptar los mismos criterios y puntos de corte supone una uniformidad en la detección de los niveles de polivictimización, no obstante, es posible que esos puntos de corte no representen adecuadamente el número de victimizaciones que realmente sufre cada grupo. Por el contrario, establecer criterios propios atendiendo a las victimizaciones reportadas por cada muestra, aporta una información más ajustada sobre la cantidad de victimizaciones experimentadas por los niños y adolescentes. Esto resulta de gran importancia si, además, tenemos en cuenta las características individuales de cada muestra, es decir, niños y adolescentes que tienen una alta vulnerabilidad y que son grupos de riesgo (p.e. ÁlvarezLister et al., 2017; Álvarez-Lister et al., 2014; Cyr et al., 2012; Guerra et al., 2016). Así como las características contextuales, que pueden llevar a la modificación de algunos ítems de los cuestionarios; por un lado, porque las definiciones legales de los delitos difieren entre países (Aho et al., 2016; Pereda et al., 2014); por otro lado, al no contemplarse determinadas victimizaciones en el lugar donde se desarrolla la investigación (Dong et al., 2017).

En resumen, más allá del método de análisis utilizado, la definición metodológica de la polivictimización va a depender de otros aspectos que van a determinar la variación de los puntos de corte y de las prevalencias halladas. 
Primero, el marco temporal que se analice. Aunque el último año de vida puede aportar datos más precisos al ser un periodo de tiempo más reciente, el periodo de vida puede aportar un perfil más completo de victimizaciones (Finkelhor et al., 2009). Segundo, la versión del instrumento que se aplica. No es lo mismo reportar un número máximo de 12 victimizaciones (versión abreviada del JVQ) que un máximo de 34 (versión original). En este punto, además, hay que añadir la variabilidad de ítems en cada estudio, ya sea por motivos contextuales u objetivos propios de la investigación, entre otros. También es crucial la persona a la que se le aplica el cuestionario. Por ejemplo, aunque la versión retrospectiva para adultos ofrece una visión completa de la toda la infancia y adolescencia, cabe la posibilidad de que no se recuerden algunos tipos de victimización o que no se informen adecuadamente. Tercero, la manera en que se contabilizan los ítems. Ya que tampoco es lo mismo contabilizar las diferentes victimizaciones sufridas en un mismo incidente (SSV), que únicamente las victimizaciones ocurridas en eventos separados (SIV), pudiendo informar de un mayor número de victimizaciones con el primer modo de puntaje.

Por todo lo expuesto, se puede afirmar que la definición metodológica de la polivictimización es diferente en cada investigación. Se resalta la necesidad de ser cautelosos a la hora de interpretar las cifras que componen el panorama epidemiológico de este fenómeno.

En esta revisión se ha pretendido realizar, desde una perspectiva metodológica, un acercamiento al concepto de polivictimización propuesto por el equipo de D. Finkelhor, seleccionando mediante unos criterios específicos una serie de artículos que forman parte de una producción científica que está en pleno crecimiento. El presente trabajo puede servir de orientación para nuevos investigadores en este campo de estudio que traten de comprender la epidemiología de la polivictimización, o que se enfrenten por primera vez a las cuestiones que plantea la ausencia de una definición metodológica.

Este estudio presenta algunas limitaciones como es la exclusión del término polyvictimization escrito con el guion en la estrategia de búsqueda. Estopuede haber dejado fuera de la revisión algunas investigaciones, ya que se usan ambos términos en lengua inglesa, polyvictimization y poly-victimization Como líneas futuras de investigación, sería

\section{Revista Española de Investigación Criminológica}

Artículo 6, Número 18 (2020)

https://doi.org/10.46381/reic.v18i1.399

www.criminologia.net

ISSN: 1696-9219 
interesante realizar un análisis comparativo del concepto de polivictimización y su prevalencia entre los estudios que utilizan el Cuestionario de Victimización Juvenil y aquellos estudios que aplican otras herramientas para detectar estos perfiles de victimización extrema.

\section{Referencias}

Aho, N., Gren-Landell, M. \& Göran, C. (2016). The Prevalence of Potentially Victimizing Events, Poly-Victimization, and Its Association to Sociodemographic Factors: A Swedish Youth Survey. Journal of Interpersonal Violence, 31(4), 620-651. doi: 10.1177/0886260514556105

Álvarez-Lister, M.S., Pereda, N., Abad, J., Guilera, G. \& GReVIA (2014). Polyvictimization and its relationship to symptoms of psychopathology in a southern European simple of adolescent outpatients. Child Abuse \& Neglect, 38, 747-756. doi: 10.1016/j.chiabu.2013.09.005

Álvarez-Lister, M.S., Pereda, N., Guilera, G., Abad, J. \& Segura, A. (2017). Victimization and Poly-Victimization in Adolescent Outpatients from Mental Health Centers: A Case-Control Study. Journal of Family Violence, 32, 197-205. doi: 10.1007/s10896016-9831-1

Bogolyubova, O., Skochilov, R. \& Smykalo, L. (2015). Childhood Victimization Experiences of Young Adults in St. Petersburg, Russia. Journal of Interpersonal Violence, 30(7), 1153-1167. doi: 10.1177/0886260514539849

Chan, K.L. (2013). Victimization and poly-victimization among school-aged Chinese adolescents: Prevalence and associations with health. Preventive Medicine, 56, 207 210. doi: 10.1016/j.ypmed.2012.12.018

Croysdale, A.E., Drerup, L.C., Bewsey, K. \& Hoffmann, N.G. (2008). Correlates of Victimization in a Juvenile Justice Population. Journal of Aggression, Maltreatment \& Trauma, 17(1), 103-117. doi:10.1080/10926770802250876

Cyr, K., Chamberland, C., Lessard, G., Clément, M.-È., Wemmers, J.-A., Collin-Vézina, D. \& Gagné, M.-H. (2012). Polyvictimization in a Child Welfare Sample of Children and Youths. American Psychological of Violence, 2(4), 385-400. doi:10.1037/a0028040

Cyr, K., Chamberland, C., Clément, M.-E., Lessard, G., Wemmers, J.-A., Collin-Vézina, D., Gagné, M.-H. \& Damant, D. (2013). Polyvictimization and victimization of children and youth: Results from a population survey. Child Abuse \& Neglect, 37, 814-820. doi: 10.1016/j.chiabu.2013.03.009

Cyr, K., Chamberland, C., Clément, M.-E., Wemmers, J.-A., Collin-Vézina, D., Lessard, G., Gagné, M.-H. \& Damant, D. (2017). The Impact of Lifetime Victimization and Polyvictimization on Adolescents in Québec: Mental Health Symptoms and Gender Differences. Violence and Victims, 32(1), 3-21. doi: 10.1891/0886-6708.VV-D-1400020

Revista Española de Investigación Criminológica

Artículo 6, Número 18 (2020)

https://doi.org/10.46381/reic.v18i1.399

www.criminologia.net

ISSN: 1696-9219 
Cyr, K., Clément, M.-E. \& Chamberland, C. (2014). Lifetime Prevalence of Multiple Victimizations and Its Impact on Children's Mental Health. Journal of Interpersonal Violence, 29(4), 616-634. doi: 10.1177/0886260513505220

Dong, F., Cao, F., Cheng, P., Cui, N. \& Li, Y. (2013), Prevalence and associated factors of poly-victimization in Chinese adolescents. Scandinavian Journal of Psychology, 54, 415-422. doi: 10.1111/sjop.12059

Finkelhor, D., Hamby, S.L., Ormrod, R.K. \& Turner, H.A (2005). The Juvenile Victimization Questionnaire: Reliability, validity, and national norms. Child Abuse \& Neglect, 29, 383-412 doi:10.1016/j.chiabu.2004.11.001

Finkelhor, D., Hamby, S.L., Turner, H.A. \& Ormrod, R.K. (2011). The Juvenile Victimization Questionnaire: 2nd Revision (JVQ-R2). Durham, NH: Crimes Against Children Research Center.

Finkelhor, D., Ormrod, R. K., \& Turner, H. A. (2007a). Polyvictimization and trauma in a national longitudinal cohort. Development and Psychopathology, 19, 149-166. doi: 10.1017/S0954579407070083

Finkelhor, D., Ormrod, R. K., \& Turner, H. A. (2007b). Polyvictimization: A neglected component in child victimization trauma. Child Abuse \& Neglect, 31, 7-26. doi: 10.1016/j.chiabu.2006.06.008

Finkelhor, D., Ormrod, R.K. \& Turner, H.A. (2009). Lifetime assessment of polyvictimization in a national sample of children and youth. Child Abuse \& Neglect, 33, 403-411. doi: 10.1016/j.chiabu.2008.09.012

Finkelhor, D., Ormrod, R.K., Turner, H.A. \& Hamby, S.L. (2005). Measuring polyvictimization using the Juvenile Victimization Questionnaire. Child Abuse \& Neglect, 29, 1297-1312. doi:10.1016/j.chiabu.2005.06.005

Finkelhor, D., Shattuck, A., Turner, H.A., Ormrod, R. \& Hamby, S.L. (2011). Polyvictimization in Developmental Context. Journal of Child \& Adolescent Trauma, 4, 291-300. doi: 10.1080/19361521.2011.610432

Ford, J.D., Chapman, J., Connor, D.F. \& Cruise, K.R. (2012). Complex Trauma and aggression in secure juvenile justice setting. Criminal Justice and Behavior, 39(6), 694-724. doi: 10.1177/0093854812436957

Ford, J.D., Elhai, J.D., Connor, D.F. \& Frueh, C. (2010). Poly-Victimization and Risk of Posttraumatic, Depressive, and Substance Use Disorders and Involvement in Delinquency in a National Sample of Adolescents. Journal of Adolescent Health, 46, 545-552. doi: 10.1016/j.jadohealth.2009.11.212

Ford, J.D., Grasso, D.J., Hawke, J. \& Chapman, J.F. (2013). Poly-victimization among juvenile justice-involved youths. Child Abuse \& Neglect, 37, 788-800. doi: 10.1016/j.chiabu.2013.01.005

Guerra, C., Inostroza, R., Villegas, J., Villalobos, L. \& Pinto-Cortez, C. (2017). Polivictimización y sintomatología postraumática: el rol del apoyo social y la autoeficacia. Revista de Piscología, 26(2), 1-10. doi: 10.5354/0719-0581.2017.47951

Guerra, C., Pereda, N., Guilera, G. \& Abad, J. (2016). Internalizing symtoms and polyvictimization in a clinical sample of adolescents: The roles of social support and non-productive coping strategies. Child Abuse \& Neglect, 54, 57-65. Doi:

Revista Española de Investigación Criminológica

Artículo 6, Número 18 (2020)

https://doi.org/10.46381/reic.v18i1.399

www.criminologia.net

ISSN: 1696-9219 
10.1016/j.chiabu.2016.03.004Hamby, S., Finkelhor, D., Ormrod, R., \& Turner, H. (2005). The Juvenile Victimization Questionnaire (JVQ): Administration and Scoring Manual. Durham, NH: Crimes Against Children Research Center.

Hamby, S., Finkelhor, D., Turner, H. \& Kracke, K. (2011). The Juvenile Victimization Questionnaire toolkit. Recuperado de http://www.unh.edu/ccrc/jvq/index_new.html

Holt, M.K., Finkelhor, D. \& Kaufman, G. (2007). Multiple victimization experiences of urban elementary school students: Associations with psychological functioning and academic performance. Child Abuse \& Neglect, 31, 503-515. doi:10.1016/j.chiabu.2006.12.006

Indias-García, S. \& De Paúl-Ochotorena, J. (2017). Lifetime victimization among Spanish adolescents. Psicotherma, 29(3), 378-383. doi: 10.7334/psicothema2016.342

Kretschmar, J. M., Tossone, K., Butcher, F., \& Flannery, D. J. (2016). Patterns of polyvictimization in a sample of at-risk youth. Journal of Child \& Adolescent Trauma, 10(4), 363-375. doi:10.1007/s40653-016-0109-9

Lätsch, D.C., Nett, J.C. \& Hümbelin, O. (2016). Poly-Victimization and Its Relationship With Emotional and Social Adjustment in Adolescence: Evidence From a National Survey in Switzerland. American Psychological Association. doi:10.1037/a0039993

Le, M.T.H., Holton, S., Nguyen, H.T., Wolfe, R. \& Fisher, J. (2015). Poly-Victimisation among Vietnamese High School Students: Prevalence and Demographic Correlates. PLOS ONE, 10(5), E0125189. doi:10.1371/journal.pone.0125189

Pereda, N. (2019). ¿Cuánta violencia es demasiada? Evaluación de la polivictimización en la infancia y la adolescencia. Papeles del Psicólogo, 40(2), 101-108. doi:10.23923/pap.psicol2019.2892

Pereda, N., Abad, J. \& Guilera, G. (2015). Victimization and Polyvictimization Among Spanish Adolescent Outpatients. Journal of Aggression, Maltreatment \& Trauma, 24, 1044-1066. doi: 10.1080/10926771.2015.1072121

Pereda, N., Abad, J. \& Guilera, G. (2017). Victimization and Polyvictimization of Spanish Youth Involved in Juvenile Justice. Journal of Interpersonal Violence, 32(21), 32723300. doi:10.1177/0886260515597440

Pereda, N., Gallardo-Pujol, D. \& Guilera, G. (2018). Good practices in the assessment of victimization: The Spanish adaptation of the Juvenile Victimization Questionnaire. Psychology of Violence, 8(1), 76-86. doi: 10.1037/vio0000075

Pereda, N., Guilera, G. \& Abad. J. (2014). Victimization and polyvictimization of Spanish children and youth: Results from a community sample. Child Abuse \& Neglect, 38, 640-649. doi: 10.1016/j.chiabu.2014.01.019

Pinto-Cortez, C., Flores-Jara, J., Pereda, N. \& Guerra, C. (2019). Victimización y polivictimización en niños, niñas y adolescents aymara y su relación con sintomatología post-traumática. INTERCIENCIA, 44(4), 229-235. Recuperado de https://www.redalyc.org/jatsRepo/339/33959119007/html/index.html

Pinto-Cortez, C., Gutiérrez-Echegoyen, P. \& Henríquez, D. (2018). Child Victimization and Polyvictimization Among Young Adults in Northern Chile. Journal of Interpersonal Violence, 1-23. doi:10.1177/0886260518759058

Revista Española de Investigación Criminológica

Artículo 6, Número 18 (2020)

https://doi.org/10.46381/reic.v18i1.399

www.criminologia.net

ISSN: 1696-9219 
Pinto-Cortez, C., Pereda, N. \& Álvarez-Lister, M.S. (2017). Child Victimization in a Community Sample of Adolescents in Northern Chile. Journal of Aggression, Maltreatment \& Trauma, 1-20. doi:10.1080/10926771.2017.1410748

Pinto-Cortez, C. \& Venegas, K. (2015). Experiencias de Victimización y Polivictimización en Jóvenes Chilenos. Señales, 9(14), 5-25. https://www.researchgate.net/publication/292148599_Experiencias de Victimizaci on y Polivictimizacion en

Reid, J. A., \& Sullivan, C. J. (2009). A latent class typology of juvenile victims and exploration of risk factors and outcomes of victimization. Criminal Justice and Behavior, XX(X), 1-24. doi:10.1177/0093854809340621

Richmond, J.M., Elliott, A.N., Pierce, T.W., Aspelmeier, J.E. \& Alexander, A.A. (2009). Polyvictimization, Childhood Victimization, and Psychological Distress in College Women. Child Maltreatment, 14(2), 12-147. doi: 10.1177/1077559508326357

Segura, A., Pereda, N., Abad J. \& Guilera, G. (2015). Victimization and polyvictimization among Spanish youth protected by the child welfare system. Children and Youth Services Review, 59, 105-112. Doi: 10.1016/j.childyouth.2015.10.011

Segura, A., Pereda, N. \& Guilera, G. (2018). Poly-victimization from different methodological approaches usgin the juvenil victimization questionnaire: Are we identifying the same victims? Journal of Trauma \& Dissociation, 19(3), 289-306. doi:10.1080/15299732.2018.1441352

Soler, L., Paretilla, C., Kirchner, T. \& Forns, M. (2012). Effectos of poly-victimization on self-esteem and post-traumatic stress symptons in Spansih adolescents. Eur Child Adolesc Psychiatry, 21, 645-653. doi:10.1007/s00787-012-0301-x

Sterzing, P.R., Ratliff, G.A., Gartner, R.E., McGeough, B.L. \& Johnson, K.C. (2017). Social Ecological Correlates of Polyvictimization among a National Sample of Transgender, Genderqueer, and Cisgender Sexual Minority Adolescents. Child Abuse \& Neglect, 67,1-12. doi: 10.1016/j.chiabu.2017.02.017

Turner, H.A., Finkelhor, D. \& Ormrod, R. (2010). Poly-Victimization in a National Sample of Children and Youth. Am J Prev Med, 38(3), 323-330. doi: 10.1016/j.amepre.2009.11.012

Turner, H.A., Finkelhor, D. \& Ormrod, R. (2006). The effect of lifetime victimization on the mental health of children and adolescents. Social Science \& Medicine, 62, 1327. doi:10.1016/j.socscimed.2005.05.030

Turner, H.A., Shattuck, A., Finkelhor, D. \& Hamby, S. (2016). Polyvictimization and Youth Violence Exposure Across Contexts. Journal of Adolescent Heatlh, 58, 208-214. doi: 10.1016/j.jadohealth.2015.09.021

\section{Agradecimientos}

Se agradece a todo el equipo del Centro de Investigación en Criminología de la Universidad de Castilla-La Mancha el apoyo y las orientaciones ofrecidas. 


\section{Financiamiento}

María Verónica Jimeno Jiménez recibe financiación del Fondo Europeo de Desarrollo Regional (FEDER) [2019/7375]

Beatriz Ortega Vidal es investigadora predoctoral en formación del Departamento de Psicología de la Universidad de Castilla-La Mancha. Es miembro del Centro de Investigación en Criminología (CIC) de la Universidad de Castilla-La Mancha, concretamente trabaja en la Línea de Investigación en Victimología (LiV). En los dos últimos años ha estado trabajando en esta línea junto con María Verónica Jimeno Jiménez, colaborando en el proyecto "Victimización de menores del sistema de protección de la infancia y del sistema de justicia juvenil en Albacete".

https://orcid.org/0000-0002-0175-9874

María Verónica Jimeno Jiménez es profesora ayudante doctora del Departamento de Psicología de la UCLM. Desde el año 2016, es miembro del Centro de Investigación en Criminología dónde dirige la Línea de Investigación en Victimología. Imparte docencia en el Grado de Criminología y en el máster de Investigación en Criminología y Delincuencia Juvenil de la UCLM. Ha publicado trabajos a nivel nacional e internacional sobre los efectos de los procesos de victimización en la infancia y la adolescencia.

iD https://orcid.org/0000-0002-6358-9444 